\title{
Obstrução intestinal devido a enterolitos em divertículo de Meckel: relato de caso*
}

\section{Small bowel obstruction caused by Meckel'S diverticulum enterolith: case report}

\author{
Ledo Mazzei Massoni Neto', Betina Bremer Hinkel', \\ Allysson Doi $^{2}$, Paulo Sérgio Martins de Alcântara ${ }^{3}$
}

\begin{abstract}
Massoni Neto LM, Hinkel BB, Dói A, Alcântara PSM. Obstrução intestinal devido a enterolitos em divertículo de Meckel: Relato de Caso. Rev Med (São Paulo). 2007 jul.-set.;86(3):155-62.

RESUMO: Divertículo de Meckel (DM) é uma patologia congênita relativamente comum, subdiagnosticada porque raramente apresenta sintomas. A maior parte dos DM sintomáticos ocorre em crianças, e se apresenta na forma de hemorragia digestiva baixa. A formação de enterolito é um evento raro no DM, pois nele raramente ocorrem as condições que se supõe necessárias à formação dos cálculos (estase e meio alcalino). A presença de enterolitos em DM está associada a óstio estreito (favorecendo estase) e ausência de mucosa gástrica (possibilitando o desenvolvimento de meio alcalino). O quadro clínico mais comum é de dor abdominal recorrente. Os achados radiológicos incluem cálculos ao RX simples de abdome e cálculos dentro de área delimitada por parede intestinal à TC. Este relato se diferencia dos anteriores por mostrar um caso de enterolito em DM com mucosa gástrica ectópica, e grande óstio comunicando o divertículo com a luz intestinal.
\end{abstract}

DESCRITORES: Dor abdominal/etiologia. Obstrução intestinal/complicações. Divertículo ileal/ patologia. Estudos de casos.

\footnotetext{
* Prêmio Oswaldo Cruz. Relato de Caso.

1. Acadêmico do $6^{\circ}$ ano da Faculdade de Medicina da USP.

2. Residente de Cirurgia Geral HCFMUSP/HU-FMUSP. Orientador.

3. Assistente do Departamento de Clínica Cirúrgica do HU-FMUSP. Orientador.

Endereço para correspondência: Ledo Mazzei Massoni Neto. Artur de Azevedo, 129. Ap. 2. Cerqueira César. CEP: 05404-010. São Paulo SP. e-mail: ledo.massoni @ gmail.com
} 


\section{INTRODUÇÃO}

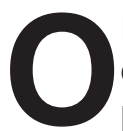

Divertículo de Meckel (DM) é anomalia congênita que resulta da persistência parcial ou total do ducto onfalo-mesentérico, normalmente reabsorvido no terceiro mês de vida fetal ${ }^{17}$. Trata-se de divertículo verdadeiro, uma vez que apresenta as três camadas da parede intestinal. Localiza-se na borda anti-mesentérica e geralmente no íleo terminal23.

Apesar de ocorrer em até $2 \%$ da população, somente $4 \%$ dos portadores apresentarão manifestações durante a vida, de forma que o distúrbio geralmente é descoberto acidentalmente, em cirurgias ou exames indicados por outras razões, ou ainda em necrópsias.

Como, em geral, não apresentam manifestações clínicas, dificilmente seu diagnóstico ocorre na ausência de complicações. Dentre elas estão a hemorragia, intussuscepção, obstrução intestinal, perfuração, diverticulites e neoplasias ${ }^{36}$.

A formação de cálculos ou enterolitos em divertículo de Meckel é um evento raro ${ }^{29}$, e a visualização deles em radiografias tem sido reportada na literatura apenas como relatos de $\operatorname{casos}^{4,6,10}$.

Episódios de suboclusão/obstrução intestinal conseqüente a enterolitíase é complicação pouco freqüente, raramente descrita e que ocorre em virtude da extrusão do enterolito para a luz do intestino delgado com obturação distal ou, mais raramente ainda, pela obstrução no nível do próprio divertículo ${ }^{19}$.

O objetivo deste relato é chamar a atenção para esta rara possibilidade diagnóstica, ressaltar os achados de exames de imagem, e descrever a possibilidade de formação de enterolitos em divertículos de Meckel com mucosa gástrica ectópica.

\section{RELATO DO CASO}

LM, 43 anos, paciente masculino, branco, casado, 2 filhas, secretário, natural e procedente de

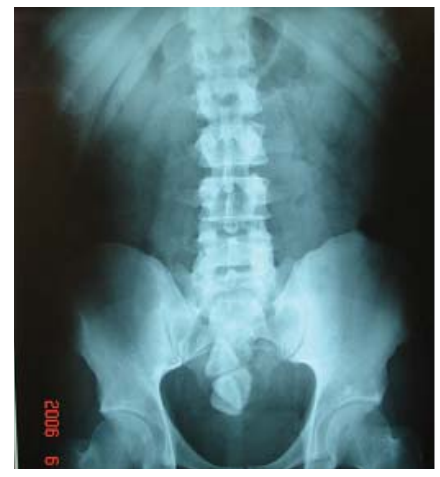

São Paulo.

Paciente conta que há 10 anos se iniciou quadro de cólicas abdominais esporádicas de leve intensidade, que melhoravam espontaneamente. Há 8 meses iniciou quadros de cólicas abdominais difusas, predominantemente em fossa ilíaca esquerda e hipogástrio, de moderada intensidade. Há 4 dias teve diminuição de eliminação e afilamento de fezes, distensão abdominal e náuseas, e há 1 dia, 5 episódios de vômitos. Negava febre, perda de peso, sangramentos, alterações de hábito urinário e não tinha antecedente de cirurgias anteriores.

Procurou o serviço de emergência do Hospital Universitário da FMUSP (01/09/05).

Ao exame físico:

- Bom estado geral, corado, desidratado +/4+, anictérico, afebril, acianótico,

- $P A=130 \times 90 \mathrm{mmHg}, \mathrm{P}=90$ b.p.m

- Cardíaco: bulhas rítmicas normofonéticas 2 tempos sem sopros

- Pulmonar: murmúrios vesiculares presentes, sem ruídos adventícios

- Abdominal: abdome plano, sem cicatrizes cirúrgicas prévias, ruídos hidroaéreos presentes, levemente distendido, sem visceromegalias, sem sinais de peritonismo, dor à palpação profunda de meso/hipogástrio e fossa ilíaca esquerda

- Toque retal com esfíncter normotônico, sem massas palpáveis ou corpos estranhos, próstata tópica

Foi submetido a uma radiografia simples de abdome, medicado com antiespasmódicos e mantido sob observação clínica.

A radiografia evidenciou 3 imagens radiopacas triangulares em região pélvica, não mostrou distensão de alças intestinais nem níveis líquidos em seu interior (Figuras 1 e 2).

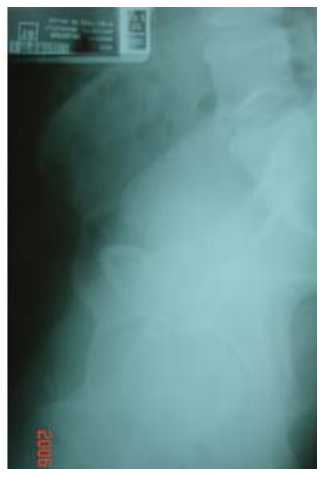

Figuras 1 e 2. Radiografias de abdome, mostrando imagens triangulares radiopacas em região pélvica. As Figuras 1 e 2 correspondem a radiografias obtidas em AP e perfil no mesmo dia (01/09/2006) 
Devido a suspeita de litíase vesical foi solicitada ultrassonografia de vias urinárias e de abdome. O resultado de ambos exames não mostrou alterações. 0 paciente apresentou melhora dos sintomas e recebeu alta, com encaminhamento para acompanhamento ambulatorial.

Dia 14/09/05 o paciente teve novas crises de cólicas abdominais e suboclusão intestinal, voltando a procurar atendimento no serviço de emergência do Hospital Universitário da FMUSP.

Seu exame físico continuava inocente, com sinais vitais normais, sem sinais de peritonismo ou obstrução intestinal. Foi submetido a nova radiografia simples de abdome (Figura 3) e subsequentemente a tomografia de abdome e pelve (Figura 4).

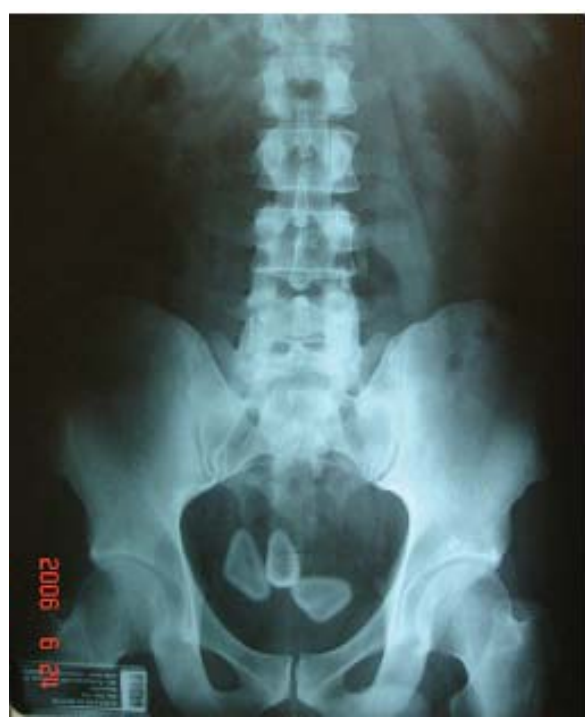

Figura 3. Radiografia de abdome, mostrando imagem triangular radiopaca em região pélvica. A Figura 3 mostra radiografia obtida no dia 14/09/05

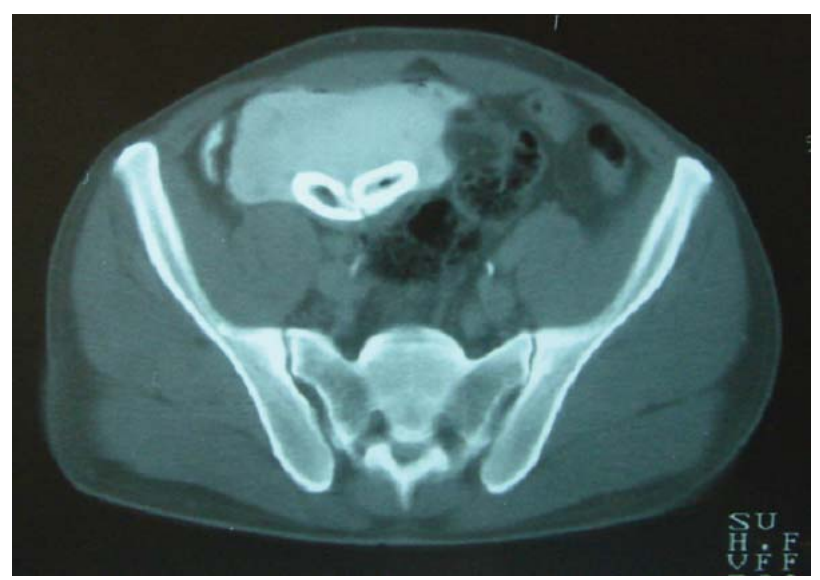

Figura 4. Tomografia Computadorizada de abdome mostrando Divertículo de Meckel com 2 enterolitos em seu interior
A radiografia novamente mostrou as imagens triangulares em topografia pélvica, sem evidências de obstrução intestinal. A tomografia computadorizada do abdome mostrou:

- Fígado de contornos e dimensões conservados, com coeficiente de atenuação normal;

- Vesícula biliar de contornos e dimensões normais;

- Ausência de dilatação de vias biliares;

- Pâncreas normal sem dilatação do ducto de Wirsung;

- Baço e rins de topografia e dimensões normais;

- Sem dilatação de sistema excretor;

- Aorta e veia cava sem alterações;

- Sem linfonodomegalias;

- Bexiga urinária de contornos lisos, morfologia conservada;

- Ausência de massas ou coleções na cavidade;

- Alças intestinais sem evidência de anormalidades, exceto por 3 imagens de cálculos no interior de alças ileais.

Novamente o paciente teve melhora importante dos sintomas após medicação sintomática com antiespasmódicos, e apresentou evacuação espontânea no hospital. Foi indicada laparotomia exploradora, não realizada pela relutância do paciente, que recebeu alta e continuou em acompanhamento ambulatorial.

Foi submetido a endoscopia digestiva alta $(12 / 05 / 05)$ a fim de se excluir qualquer sinal de fístula colecisto gástrica ou duodenal (íleo-biliar). Este exame também não evidenciou alterações.

O paciente continuou assintomático em seu acompanhamento, foi submetido a colonoscopia na tentativa de alcançar os enterolitos sem procedimento cirúrgico.

Realizado exame dia 02/02/06, com introdução do colonoscópio até $25 \mathrm{~cm}$ do íleo terminal, visualizadas erosões rasas ladeadas por mucosa íntegra, que foram biopsiadas. Não foram observadas imagens de cálculos. Ceco, cólon ascendente, transverso, descendente, sigmóide e reto não mostravam alterações.

O resultado do exame anátomo patológico das lesões em íleo distal foram de erosões reparadas e hiperplasia linfóide reativa em mucosa entérica.

Em maio de 2006, o paciente iniciou quadro de cólicas abdominais mais intensas sendo proposta a retirada de enterolitos por procedimento cirúrgico.

Em 19/06/06 o paciente foi internado na enfermaria da Clínica Cirúrgica do Hospital Universitário da FMUSP em caráter eletivo.

Ao exame físico: 
- Bom estado geral, corado, hidratado, anictérico, afebril, acianótico;

- $P A=130 \times 80 \mathrm{mmHg} P=68$ b.p.m;

- Cardíaco: bulhas rítmicas normofonéticas 2 tempos sem sopros;

- Pulmonar: murmúrios vesiculares simétricas sem ruídos adventícios;

- Abdominal: RHA presentes, sem visceromegalias, sem sinal de peritonismo, dor à palpação profunda de fossa ilíaca esquerda e meso/ hipogástrio;

- Extremidades sem alterações;

- Toque retal sem alterações.

Dia 20/06/06 foi submetido a cirurgia (Figuras $6,7,8,9$ e 10):

- Laparotomia mediana iniciando $4 \mathrm{~cm}$ acima do umbigo até pube;
- Inventário da cavidade:

- Observado várias aderências entre diafragma e fígado, entre baço e diafragma;

- Aderências entre alças de delgado;

- Fibrose em alguns pontos do meso de delgado;

- Não foram encontrados cálculos à palpação de vesícula biliar;

- Não foi observado nenhum tipo de fístula colecisto duodenal;

- Identificada formação sacular na borda contramesenterial da alça, compatível com divertículo de Meckel, a $35 \mathrm{~cm}$ da válvula íleo-cecal;

- A palpação desta estrutura era sugestiva da presença de enterolitos em seu interior;

- Optado por realização de enterectomia segmentar de aproximadamente $15 \mathrm{~cm}$ de extensão;

- Reconstrução do trânsito realizado com anastomose término terminal manual em 2 planos;

- Sutura do plano seromuscular com algodão 3-0 pontos separados e plano total com vicryl 3-0 pontos contínuos;

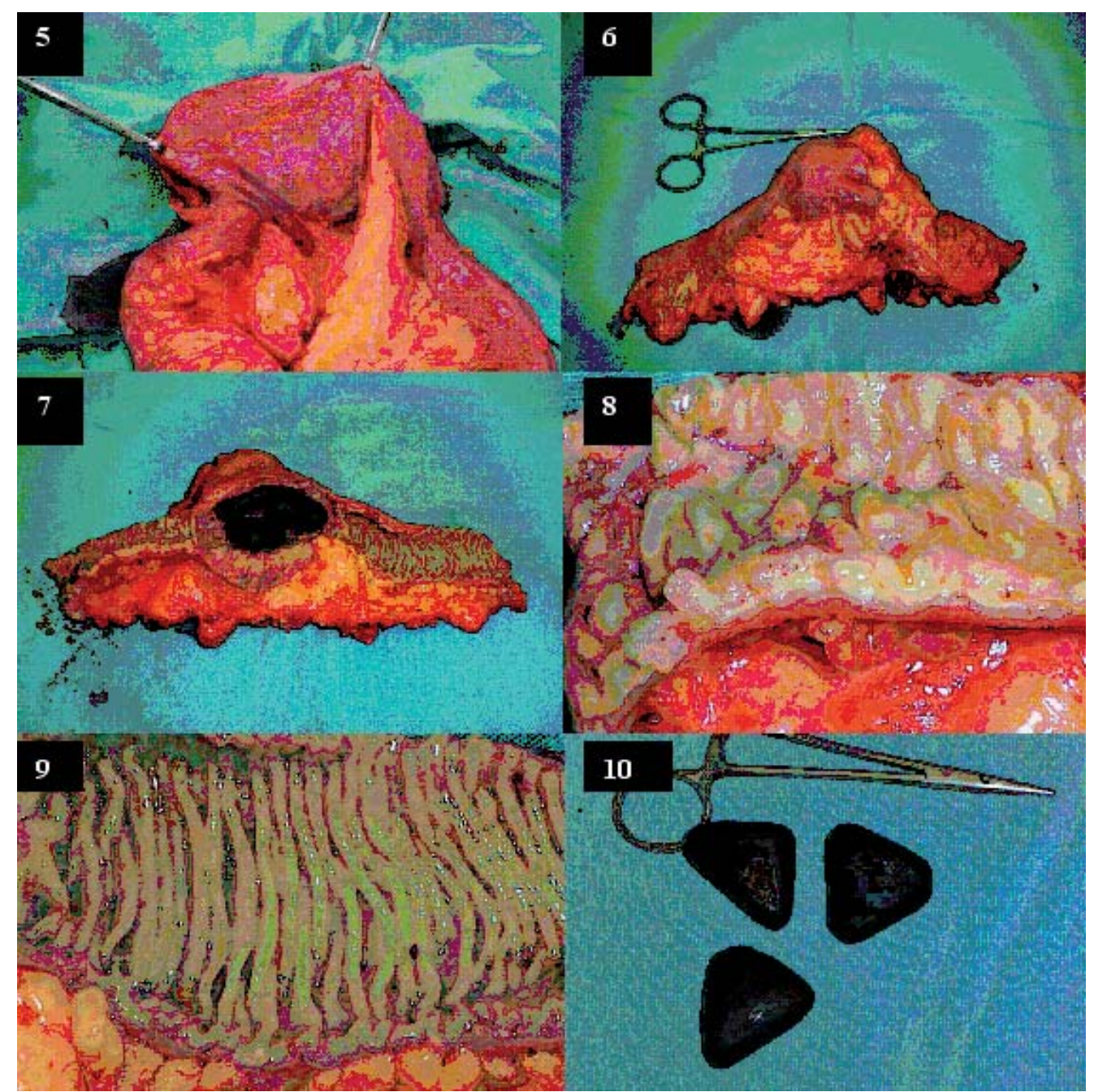

Figuras 5, 6, 7, 8, 9,10. Mostrando, respectivamente, o divertículo antes de ser ressecado (5); a peça ressecada (6); abertura da peça (7); mucosa gástrica ectópica (8); mucosa intestinal (9); enterolitos (10) 
Paciente evoluiu bem sem complicações no pós operatório, sem intercorrências. Iniciado dieta no $4^{\circ}$ pós operatório, apresentou evacuação no mesmo dia. Recebeu alta hospitalar no $5^{\circ}$ pós operatório em bom estado geral e sem queixas.

O exame anátomo patológico da peça concluiu: divertículo de Meckel com mucosa antral e fúndica, enterite crônica moderada sem sinal de malignidade e cálculos compatíveis com enterolito.

\section{DISCUSSÃO}

O divertículo de Meckel é um divertículo intestinal verdadeiro, que resulta da falha de obliteração do ducto onfalo-mesentérico, ou vitelino, durante o terceiro mês de vida fetal ${ }^{17,32}$. Falhas nesta etapa podem se apresentar de várias formas: pode haver permanência do ducto totalmente patente, comunicando a parte terminal do íleo ao umbigo (fístula êntero-umbilical), pode haver persistência somente da porção junto ao umbigo (seio umbilical), ou da porção média (cisto vitelino ou cisto do ducto onfalomesentérico), ou a persistência de somente um cordão fibroso unindo o íleo ao umbigo, e finamente a permanência da porção proximal ao intestino constitui o divertículo de Meckel, que é a modalidade mais comum do espectro das falhas de obliteração e absorção do ducto onfalo-mesentérico $(98 \%)^{18}$.

Apresenta todas as camadas intestinais e, em aproximadamente $50 \%$ dos casos, possui tecidos ectópicos ${ }^{21}$. Dentre esses destacam-se os de origem gástrica (correspondente a $60-85 \%$ ) e pancreático $(5-16 \%)^{7,20}$. Fabricus Hildanus foi o primeiro a relatar um caso em 1598, e Johann Meckel o primeiro a descrever a anatomia e embriologia desse divertículo em 1809.

É a anormalidade congênita mais comum no trato gastrointestinal, ocorrendo em aproximadamente $2 \%$ da população ${ }^{14}$. Localiza-se na borda anti-mesentérica do íleo e em $99 \%$ das vezes está a até $90 \mathrm{~cm}$ da válvula ileocecal, embora haja relatos de divertículos a $180 \mathrm{~cm}^{35}$. Geralmente se apresenta como um divertículo curto de base $\operatorname{larga}^{28} \mathrm{e}$ tem seu suprimento de sangue proveniente de um ramo terminal da artéria mesentérica superior, que cruza o íleo até o divertículo ${ }^{8,20,35}$.

O divertículo é assintomático na maioria da população e seu diagnóstico é feito geralmente em laparotomias e laparoscopias indicadas por outros motivos $^{14}$. A literatura diverge muito a respeito da taxa de complicação dos divertículos, porém estimase em $4,2 \%$ a probabilidade da doença se tornar sintomática em algum momento da vida ${ }^{30}$. Apesar desta afecção atingir igualmente ambos os sexos ${ }^{1,35}$, é mais freqüentemente sintomático em indivíduos do sexo masculino, o que causa maior taxa de diagnóstico neste grupo ${ }^{1,20}$.

As complicações se manifestam como hemorragias, intussuscepção, inflamação, perfuração, obstrução, volvo, neoplasias e enterolitos ${ }^{22}$. Sangramento é a complicação mais freqüente, especialmente em crianças abaixo dos 2 anos, devido a ulceração por mucosa gástrica heterotópica ${ }^{2,9,25}$. Já em adultos, muitos se apresentam com quadros inflamatórios e obstrução intestinal, tendo como mecanismos intussuscepção, bridas inflamatórias, volvo, hérnias internas, hérnias de Littré, torção de pedículo, enterolitos ou fitobezoares ${ }^{22}$.

A formação de cálculos ou enterolitos no interior de divertículos de Meckel é um evento extremamente raro ${ }^{19,22}$. Na literatura esse evento só é descrito em formas de relato ou série de casos, sempre com poucos pacientes. Kussumoto et al. estudaram a prevalência de complicações em 776 pacientes com o diagnóstico de divertículo de Meckel e encontraram enterolitos em apenas 2 desses pacientes ${ }^{16}$. Damien Grinsell e Donaldson em outro relato de caso levantaram 60 casos descritos em toda literatura12. Já "The Armed Forces Institute of Pathology" relatou incidência de $10 \%$ em sua casuística ${ }^{27}$.

Enterolitos são relativamente comuns em divertículos do intestino grosso, possivelmente devido ao meio alcalino e baixa motilidade destes divertículos (geralmente falsos divertículos, sem camada muscular e, portanto, sem motilidade), e raros em divertículos de Meckel, uma vez que estas condições (estase e pH alcalino) não ocorrem na maioria nestes divertículos. A ausência de mucosa gástrica ectópica nos divertículos de Meckel em que foram relatados enterolitos sugere que esta condição possibilite o desenvolvimento de ambiente alcalino, 0 que favorece a precipitação de sais minerais (principalmente sais de cálcio) $)^{12,27}$, e a estase no interior do divertículo de Meckel parece ser decorrente de deficiência do peristaltismo local (divertículo com camada muscular pouco desenvolvida), ou obstrução do óstio de comunicação do divertículo com a luz ileal por edema secundário a inflamação (diverticulite), ou ainda por um pedículo estreito ${ }^{17,22,27,29,33}$. Além de favorecer o desenvolvimento de $\mathrm{pH}$ alcalino, a ausência de mucosa gástrica aumenta a chance de o divertículo permanecer assintomático durante muito tempo, possibilitando a precipitação de cálcio e outros minerais envolvidos na formação dos enterolitos ${ }^{27}$. Corpos estranhos, como sementes, no 
interior do divertículo, podem se tornar o núcleo de formação do cálculo. Provavelmente devido a sua natureza crônica, enterolitos são mais freqüentemente descritos em adultos.

Em nosso relato foram observados dois achados inusitados. O primeiro referente a presença de mucosa ectópica gástrica fúndica e antral no interior do divertículo, pois, como já mencionado a grande maioria se forma em ambiente alcalino. O segundo, foi a presença de base larga, fato que não contribui para formação de um ambiente de estase do conteúdo intestinal.

As complicações de enterolitíase consistem em obstrução de intestino delgado, por extrusão dos cálculos para a luz intestinal, sangramento, e abdome agudo por diverticulite e perfuração. Obstrução de delgado por enterolito é uma complicação rara, clinicamente semelhante a íleo biliar ${ }^{11}$.

A radiografia simples de abdome revelou $88 \%$ dos enterolitos de divertículo de Meckel em estudo restrospectivo, sendo que desses $57 \%$ se localizavam em fossa ilíaca direita, 14\% em fossa ilíaca esquerda, $14 \%$ em região pélvica central e 14\% em quadrante superior direito do abdome ${ }^{27}$. A mobilidade do(s) enterolito(s) com a mudança de decúbito pode auxiliar no diagnóstico ${ }^{5}$. Além disso, a radiografia simples de abdome é importante no diagnóstico de obstrução intestinal independente de sua etiologia.

Em nosso relato foi observada a mobilidade dos cálculos com mudança postural, porém somente com a radiografia de abdome não se podiam excluir as possibilidades de litíase vesical ou íleo biliar.

A ultrassonografia abdominal também pode ser um importante exame na investigação de cálculos intestinais, seja pela possibilidade de se fazer o diagnóstico propriamente dito, como também avaliar a vesícula biliar e vias biliares na procura de cálculos, aerobilia, fístulas bilio-digestivas a fim de se afastar a hipótese de íleo biliar22.

Ultrassonografia e tomografia computadorizada de abdome são exames mais sensíveis que a radiografia simples para detecção de calcificações, mas pela raridade dos enterolitos em divertículos de Meckel, poucos foram os estudos relatados na literatura onde os diagnósticos foram feitos por tais exames $^{27,31}$.

De todos os exames citados, certamente a tomografia computadorizada é o exame com maior acurácia no diagnóstico de divertículos intestinais contendo cálculos em seu interior ${ }^{15}$, além de ter a possibilidade de mostrar complicações como dilatação de alças, massas abdominais, fístulas, abscessos e diagnosticar cálculos em outras topografias.

Com relação ao caso apresentado a ultrassonografia de abdome não diagnosticou a enterolitíase, porém pôde nos dar informações importantes como ausência de cálculos vesicais e vesícula biliar sem alterações, excluindo assim algumas das hipóteses diagnósticas levantadas ao se observar a radiografia simples do abdome. Por outro lado a tomografia de abdome total firmou o diagnóstico de enterolitíase sem complicações, mostrando inclusive o número de cálculos.

A conduta frente a casos de divertículos de Meckel encontrados acidentalmente em laparotomias é controversa. Há diversos autores que recomendam sua ressecção, pois alegam que é impossível macroscopicamente definir que não haja mucosa heterotópica no divertículo ou que ele apesar de ter base larga não venha a ter algum tipo de complicação no futuro ${ }^{3,26}$.

Outros autores alegam que para devido a baixa probabilidade do divertículo vir a ser sintomático $(4,2 \%)$ seriam necessárias aproximadamente 800 diverticulectomias para salvar a vida de um único doente. Além disso, há de se levar em conta as mortalidades e morbidades que o procedimento acarreta $^{13,24}$.

Quando o divertículo se torna sintomático é consenso na literatura seu tratamento cirúrgico. Porém ainda restam dúvidas quanto à operação empregada: diverticulectomia ou enterectomia ${ }^{34}$.

Nos casos de enterolitos em divertículos de Meckel, quando existe a extrusão do cálculo para a luz intestinal com impactação distal pode-se tentar a realização de ordenha distal em direção ao ceco. Caso não seja possível realiza-se enterotomia com retirada em local com menos inflamação ${ }^{19}$. As ressecções intestinais deverão ser realizadas quando existirem sinais de inflamação, necrose, perfuração intestinal, ou formação de abscesso peri-diverticular ${ }^{22}$.

A conduta no caso relatado foi conforme a literatura preconiza, sendo realizado enterectomia segmentar tendo em vista sinais de complicação locais como aderências e inflamação crônica.

Em conclusão, pode-se dizer que enterolito em divertículo de Meckel é uma complicação raríssima, mas que deve ser incluído no diagnóstico diferencial de suboclusão e obstrução intestinal. Principalmente nos casos onde-se pode observar cálculos abdominais em regiões não usuais, ou móveis conforme o decúbito. 
Massoni Neto LM, Hinkel BB, Dói A, Alcântara PSM. Small bowel obstruction caused by Meckel's diverticulum enterolith: case report. Rev Med (São Paulo). 2007 jul.-set.;86(3):155-62.

\begin{abstract}
Meckel Diverticulum (MD) is a relatively common congenital anomaly, but is rarely diagnosed because it is usually asymptomatic. Most of the symptomatic MD presents as intestinal bleeding in children. Enterolith is a rare complication in MD, because the conditions supposed to be necessary for the stones formation (stasis and alkaline environment) are uncommon. Enteroltith formation have been reported in those MD with narrow neck (witch contributes to stasis) and absence of ectopic gastric mucosa (witch allows the development of a alkaline environment). The most common symptom is recurrent abdominal pain. The image findings are stones seen in the plain films or in the interior of the intestine wall at CT. This report is different from the previous by showing enteroliths in a MD with ectopic gastric mucosa and a wide neck communicating the diverticulum to the intestinal lumen.
\end{abstract}

KEY WORDS: Abdominal pain/etiology. Intestinal obstruction/complications. Meckel diverticulum/patology. Case studies.

\title{
REFERÊNCIAS
}

1. Arnold JF, Pellicane JV. Meckel's diverticulum: a ten-year experience. Am Surg. 1997;63:354-5.

2. Artigas V, Calabuig R, Badia F, Rius X, Allende L, Jover J. Meckel's diverticulum: value of ectopic tissue. Am J Surg. 1986;151:631-4.

3. Aubrey AD. Meckel's diverticulum. Arch Surg. 1970;100:144-6.

4. Benhamou G. Small intestinal obstruction by enterolith from Meckel's diverticulum. Int Surg. 1979;64:43-5.

5. Brettner A, Eupharat EJ. Radiol significance of primary enterolithiasis. Radiology. 1970;94:283-8.

6. Christiansen KH, Cancelmo RP. Meckel's stone ileus. AJR. 1967;99:139-41.

7. Cullen JJ, Kelly KA, Moir CR, Hodge DO, Zinsmeister AR, Melton LJ 3. Surgical management of Meckel's diverticulum. An epidemiologic, population-based study. Ann Surg. 1994;220:564-9.

8. D'Souza CR, Prokopishyn H. Axial volvulus of small bowel caused by Meckel's diverticulum. Surgery. 1993;114:984-7.

9. DiGiacomo JC, Cottone FJ. Surgical treatment of Meckel's diverticulum. South Med J. 1993;86:671-5.

10. Feldman MI. Calculi in Meckel's diverticulum. Radilogy. 1966;86:541-3.

11. Grant ABF. Meckel stone ileus: a case report. Aust $N$ Z J Surg. 1981;51:77-8.

12. Grinsell D, Donaldson E. Giant Meckel's diverticulum whith enterolith formation. Aust N Z J Surg. 2003;73:968-9.

13. Hedenberg C. Surgical indications in Meckel's diverticulum. Acta Chir Scand. 1969;135:530-3.

14. Justus PG, Bergman JJ, Reagan TR. Enteroliths in a Meckel's diverticulum mimicking gallstone ileus. J Fam Pract. 1987;24:299-300.

15. Kornprat $P$, Langner $C$, Mischinger HJ. Enterolithiasis in jejunal diverticulosis, a rare cause of obstruction of small intestine: a case report. Wien Klin Wochenschr. 2005;117:297-9

16. Kussumoto $H$, Yoshida $M$, Takahashi I, Anai $H$, Maehara Y, Sugimachi K. Complications and diagnosis of Meckel's diverticulum in 776 patients. Am J Surg. 1992; 164:382-3.

17. Lemos R, Binato M. Enterolitíase em paciente com divertículo de Meckel. J Bras Med. 1994;66:93-4.

18. Levy $A D$, Hobbs $C M$, Meckel. Diverticulum: radiologic features with pathologic correlation. RadioGraphics. 2004;24:565-87.

19. Lopez PV, Welch JP. Enterolith intestinal obstruction owing to acquired and congenital diverticulosis. Report of two cases and review of literature. Dis Colon Rectum. 1991;34:941-4.

20. Mackey WC, Dineen P. A fifty-year experience whith Meckel's diverticulum. Surg Gynecol Obstet. 1983;156:56-64.

21. Martin JP, Connor PD, Charles K. Meckel's diverticulum. Am Fam Physician. 2000;61:1037-42,1044.

22. Martinez CAR, Priolli DG, Palma RT, Wasisberg J, Margarido NF. Obstrução intestinal por enterolito formado em divertículo de Meckel: relato de caso. Rev Bras Coloproctol. 2004;24:165-9.

23. Martins MVDC, Duarte JGC, Martins HS. Tratamento videolaparoscópico da hemorragia digestiva por divertículo de Meckel. Rev Bras Videocir. 2004;2(1):28-30.

24. Matsagas MI, Fatouros M, Koulouras B, Giannoukas AD. Incidence, complications, and management of Meckel's diverticulum. Arch Surg. 1995;13:143-6.

25. Meckel JF. Handbuch der pathologischen anatomie, v.1. Leipzig: Reclam; 1812.

26. Michas CA, Cohen SE, Wolfman EF. Meckel's diverticulum: should it be excised incidentally at operation? Am J Surg. 1975;129:682-5.

27. Pantongrag-Brown L, Levine MS, Buetow PC, Buck JL, 
Elsayed AM. Meckel's enterolith: clinical, radiologic, and pathologic findings. Am J Roentgenol. 1996;167:144750.

28. Rutherford RB, Akers DR. Meckel's diverticulum; a review of 148 pediatric patients, with special reference to the pattern of bleeding and mesodiverticular vascular bands. Surgery. 1966;59:618-26.

29. Sharma G, Benson CK. Enteroliths in Meckel's diverticulum: report of a case and review of the literature. Can J Surg. 1970;13:54-8.

30. Soltero MJ, Bill AH. The natural history of Meckel's diverticulum and its relation to incidental removal. Am J Surg. 1976;32:168-73.

31. Torii Y, Hisatsume I, Imamura K, Morita K, Kumagaya N, Nakata H. Giant Meckel diverticulum containing enteroliths diagnosed by computed tomography and sonography. Gastrointest Radiol. 1989;14:167-9.

32. Turgeon DK, Barnett JL. Meckel's diverticulum. Am J Gastroenterol. 1990;85:777-81.

33. Vans Es HW, Sybrandry R. Diagnoses please. Case 19: enteroliths in a Meckel's diverticulum. Radiology. 2000;214:524-6.

34. Vasquez JC, Lee SH, Coimbra R, Hoyt DB. Small bowel obstruction caused by a Meckel's diverticulum enterolith. Internet J Surg. 2002;03(2).

35. Willians RS. Management of Meckel's diverticulum. $\mathrm{Br}$ J Surg. 1981;68:477-80.

36. Yahchouchy EK, Marano AF, Etienne JC, Fingerhut AL. Meckel's diverticulum. J Am Coll Surg. 2001;192:65862. 